STRUCTURAL SCIENCE CRYSTAL ENGINEERING MATERIALS

ISSN 2052-5206

Received 28 September 2020

Accepted 2 February 2021

Edited by J. Lipkowski, Polish Academy of Sciences, Poland

Keywords: rare earth intermetallic; magnetic structure; symmetry analysis; neutron diffraction.

Supporting information: this article has supporting information at journals.iucr.org/b

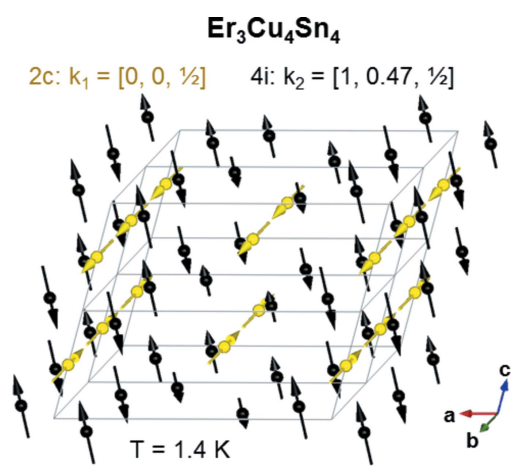

(c) () OPEN OP ACCESS

\section{Symmetry analysis of complex magnetic structure in monoclinically distorted $\mathrm{Er}_{3} \mathrm{Cu}_{4} \mathrm{Sn}_{4}$}

\author{
Stanisław Baran, ${ }^{\mathrm{a} *}$ Aleksandra Deptuch, ${ }^{\mathrm{b}}$ Bogusław Penc, ${ }^{\mathrm{a}}$ Andreas Hoser ${ }^{\mathrm{c}}$ and \\ Andrzej Szytuła ${ }^{a}$
}

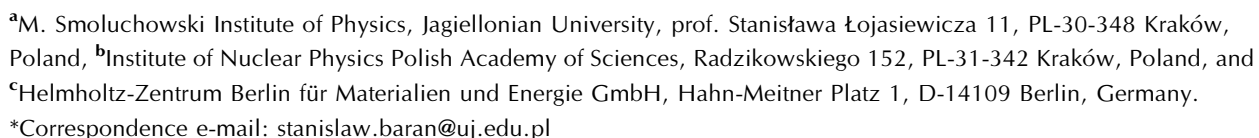

The magnetic structure in $\mathrm{Er}_{3} \mathrm{Cu}_{4} \mathrm{Sn}_{4}$ has been determined using high-resolution powder neutron diffraction, supported by symmetry analysis. At low temperatures, $\mathrm{Er}_{3} \mathrm{Cu}_{4} \mathrm{Sn}_{4}$ assumes a crystal structure of the $\mathrm{Tm}_{3} \mathrm{Cu}_{4} \mathrm{Sn}_{4}$ type (in the monoclinic space group $C 2 / m$ ). The Er atoms occupy two distinct Wyckoff sites: $2 c$ and $4 i$. It has been found that the Er magnetic moments on the $2 c$ site form a commensurate antiferromagnetic structure $\left(\mathbf{k}_{1}=\left[0,0, \frac{1}{2}\right]\right)$ below $6 \mathrm{~K}$. The magnetic moments reach $8.91(8) \mu_{\mathrm{B}}$ at $1.4 \mathrm{~K}$ and are parallel to the $b$ axis. The Er magnetic moments on the $4 i$ site order below $2 \mathrm{~K}$ and form an incommensurate antiferromagnetic sine-modulated structure $\left(\mathbf{k}_{2}=[1\right.$, 0.4667 (1), $\left.\frac{1}{2}\right]$ ), with magnetic moments lying in the $a c$ plane and perpendicular to the $a$ axis. The amplitude of modulation equals 8.7 (1) $\mu_{\mathrm{B}}$ at $1.4 \mathrm{~K}$.

\section{Introduction}

The $R_{3} T_{4} X_{4}$ family of compounds, where $R$ is a rare earth element, $T$ is a transition metal (i.e. an element with a partially filled $d$-electron sub-shell)] and $X$ is $\mathrm{Si}, \mathrm{Ge}$ or $\mathrm{Sn}$, has been investigated extensively for decades. At room temperature (RT), the majority of these compounds have an $\mathrm{Gd}_{3} \mathrm{Cu}_{4} \mathrm{Ge}_{4^{-}}$ type crystal structure (in the orthorhombic space group Immm, No. 71) (Rieger, 1970; Hanel \& Nowotny, 1970), with two formula units per unit cell. The $R$ atoms occupy two crystallographic sites, i.e. $2 d$ and $4 e$, the transition metal $(T)$ occupies the $8 n$ site, while the $X$ atoms occupy the $4 f$ and $4 h$ sites. Only select representatives of the $R_{3} \mathrm{Cu}_{4} \mathrm{Sn}_{4}$ family [those with $R=$ Tm (Thirion et al., 1983) or Lu (Romaka et al., 2008)] assume a structure of the $\mathrm{Tm}_{3} \mathrm{Cu}_{4} \mathrm{Sn}_{4}$-type, related to the monoclinic space group $C 2 / m$ (No. 12), already at room temperature. Two other compounds, namely, $R_{3} \mathrm{Cu}_{4} \mathrm{Sn}_{4}(R=$ Ho and Er), show a structural phase transition from the orthorhombic to the monoclinic structure with decreasing temperature. The transition temperatures are $62(2)$ and 262 (2) K for $R=$ Ho and Er, respectively (Cadogan et al., 2014). The monoclinic structure of the $\mathrm{Tm}_{3} \mathrm{Cu}_{4} \mathrm{Sn}_{4}$-type can be described in either the space group $C 2 / \mathrm{m}$ or $I 2 / \mathrm{m}$. In the first description, which is used in this work, the rare earth atoms occupy the $2 c$ and $4 i$ sites, while each of the two remaining elements $(T$ and $X)$ resides on two $4 i$ sites.

A summary of the magnetic properties of the $R_{3} T_{4} X_{4}$ compounds has been reported previously (Ryan et al., 2010; Szytuła et al., 2007). Most of the compounds are antiferro- 
magnets (AF) at low temperature. Also, the $\mathrm{Er}_{3} \mathrm{Cu}_{4} \mathrm{Sn}_{4}$ investigated in this work is an antiferromagnet at low temperature. Macroscopic magnetic data show either three maxima [at $2.2 \mathrm{~K}\left(\chi^{\prime \prime}\right)$, as well as at 3.6 and $5.8 \mathrm{~K}\left(\chi^{\prime}\right)$; Ryan et al., 2004] or a broad maximum between 2.0 and $3.6 \mathrm{~K}$ and a distinct one at $6.0 \mathrm{~K}$ (Wawrzyńska et al., 2003). Temperature dependence of the ${ }^{119} \mathrm{Sn}$ hyperfine fields (Ryan et al., 2004), together with the intensities of Bragg reflections of magnetic origin (Wawrzyńska et al., 2003), imply a single transition at $6.0 \mathrm{~K}$. Interpretation of the previous neutron diffraction data, based on the orthorhombic crystal structure, led to magnetic order described by the propagation vector $\mathbf{k}_{1}=\left[\frac{1}{2}, \frac{1}{2}, 0\right]$ on the $2 d$ site and $\mathbf{k}_{2}=[0,0,0.08]$ on the $4 e$ one, below $6.0 \mathrm{~K}$ (Wawrzyńska et al., 2003). However, such a model of the magnetic structure was characterized by relatively large values of the reliability factors $R_{\text {magn }}$ of 21.61 and $22.99 \%$ for the $2 d$ and $4 e$ sites, respectively.

This work reports new studies of the $\mathrm{Er}_{3} \mathrm{Cu}_{4} \mathrm{Sn}_{4}$ magnetic structure which have been performed using the E6 and E9 diffractometers installed at the BERII reactor in HelmholtzZentrum Berlin. Both instruments are equipped with highefficiency position-sensitive area detectors. Furthermore, the latter is a high-resolution instrument. These features made it possible to confirm that $\mathrm{Er}_{3} \mathrm{Cu}_{4} \mathrm{Sn}_{4}$ assumes, in fact, the monoclinic variant of the crystal structure. Moreover, the thermal evolution of the magnetic order indicates that the magnetic moments on the $2 c$ and $4 i$ sites order at different critical temperatures.
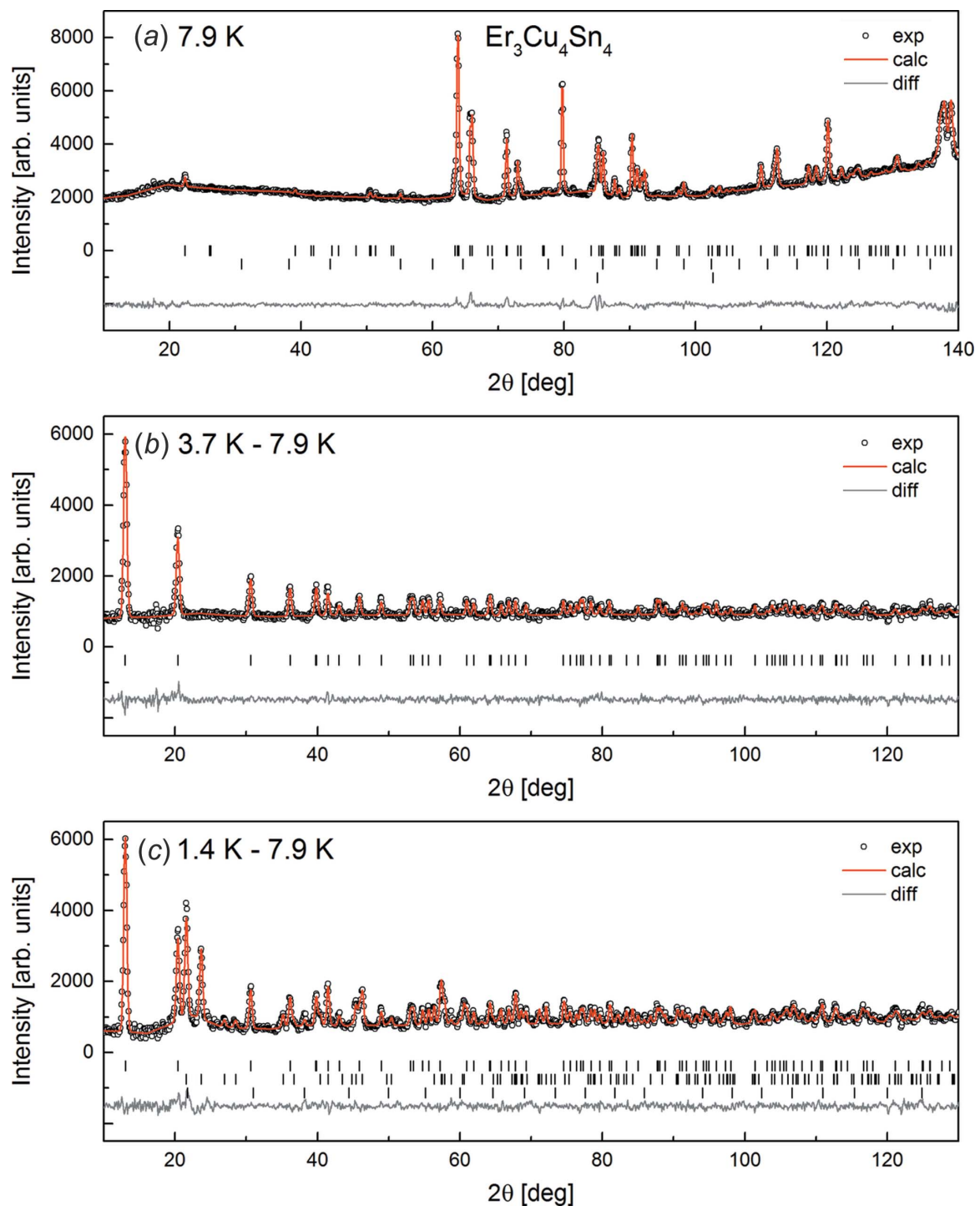

Figure 1

Neutron diffraction patterns of $\mathrm{Er}_{3} \mathrm{Cu}_{4} \mathrm{Sn}_{4}$, collected on an E9 high-resolution diffractometer with $\lambda=2.815 \AA$, at (a) $7.9 \mathrm{~K}$ (the paramagnetic state), together with the difference patterns showing pure magnetic contributions, $(b) 3.7-7.9 \mathrm{~K}$ and $(c)$ 1.4-7.9 K. The open circles represent the experimental points. The solid lines indicate the calculated profiles and the difference between the obtained and calculated intensity (at the bottom of each diagram). The vertical bars indicate the positions of Bragg reflections $(a)$ of nuclear origin corresponding to the main $\mathrm{Er}_{3} \mathrm{Cu}_{4} \mathrm{Sn}_{4} \mathrm{phase}($ first row) and to the impurity phases $\mathrm{Er}_{2} \mathrm{O}_{3}$ (second row) and $\mathrm{Cu}$ (third row), and those of magnetic origin corresponding to $(b)$ the ordering of the Er magnetic moments occupying the $2 c$ site and $(c)$ the ordering of the Er magnetic moments occupying the $2 c$ (first row) and $4 i$ (second row) sites and the antiferromagnetic order in $\mathrm{Er}_{2} \mathrm{O}_{3}$ (third row). 
Table 1

Crystallographic data for $\mathrm{Er}_{3} \mathrm{Cu}_{4} \mathrm{Sn}_{4}$ obtained from Rietveld refinement, based on the high-resolution neutron powder diffraction patterns collected at 7.9, 3.7 and $1.4 \mathrm{~K}$ (E9 diffractometer, $\lambda=2.815 \AA$ ).

$R_{\text {Bragg }}$ and $R_{F}$ refer to the residuals reporting fit quality. $\mathrm{NN}$ indicates the distance between the nearest neighbours and NNN refers to the next nearest neighbours.

\begin{tabular}{llll}
\hline$T(\mathrm{~K})$ & 7.9 & 3.7 & 1.4 \\
$a(\AA)$ & $16.0842(7)$ & $16.0831(6)$ & $16.0845(6)$ \\
$b(\AA)$ & $4.3889(2)$ & $4.3887(2)$ & $4.3889(2)$ \\
$c(\AA)$ & $6.8732(4)$ & $6.8732(3)$ & $6.8734(3)$ \\
$\beta\left({ }^{\circ}\right)$ & $115.757(3)$ & $115.754(3)$ & $115.751(3)$ \\
$V\left(\AA^{3}\right)$ & $436.98(4)$ & $436.95(3)$ & $437.03(3)$ \\
$x_{\mathrm{Er} 2}$ & $0.130(1)$ & $0.129(1)$ & $0.130(1)$ \\
$z_{\mathrm{Er} 2}$ & $0.118(3)$ & $0.117(3)$ & $0.119(3)$ \\
$x_{\mathrm{Cu} 1}$ & $0.322(1)$ & $0.321(2)$ & $0.322(1)$ \\
$z_{\mathrm{Cu} 1}$ & $0.518(3)$ & $0.518(3)$ & $0.515(3)$ \\
$x_{\mathrm{Cu} 2}$ & $0.338(2)$ & $0.338(2)$ & $0.338(2)$ \\
$z_{\mathrm{Cu} 2}$ & $0.153(3)$ & $0.152(3)$ & $0.156(3)$ \\
$x_{\mathrm{Sn} 1}$ & $0.215(2)$ & $0.216(2)$ & $0.216(2)$ \\
$z_{\mathrm{Sn} 1}$ & $0.728(4)$ & $0.731(4)$ & $0.728(4)$ \\
$x_{\mathrm{Sn} 2}$ & $0.505(2)$ & $0.506(2)$ & $0.507(2)$ \\
$z_{\mathrm{Sn2}}$ & $0.210(3)$ & $0.208(3)$ & $0.211(3)$ \\
$\mathrm{NN}(\operatorname{Er} 2-\operatorname{Er} 2)(\AA)$ & $3.77(2)$ & $3.74(2)$ & $3.77(2)$ \\
$\mathrm{NNN}(\mathrm{Er} 1-\mathrm{Er} 2)(\AA)$ & $3.83(2)$ & $3.82(2)$ & $3.84(2)$ \\
$R_{\mathrm{Bragg}}(\%)$ & 7.99 & 6.65 & 5.94 \\
$R_{F}(\%)$ & 7.68 & 5.10 & 4.30
\end{tabular}

\section{Results}

\subsection{Crystal structure}

The neutron diffraction pattern, collected at $7.9 \mathrm{~K}$ on the E9 high-resolution diffractometer for $\mathrm{Er}_{3} \mathrm{Cu}_{4} \mathrm{Sn}_{4}$ in the paramagnetic state [Fig. 1(a)], unambiguously confirms a monoclinic crystal structure of the $\mathrm{Tm}_{3} \mathrm{Cu}_{4} \mathrm{Sn}_{4}$-type. While considering the $C 2 / m$ space group, the atoms occupy the following Wyckoff sites: Er at $2 c\left(0,0, \frac{1}{2}\right)$ and $4 i(x, 0, z)$, while each of the remaining elements $(\mathrm{Cu}$ and $\mathrm{Sn})$ resides at two different $4 i$ sites $(x, 0, z)$ (see Fig. 2$)$. The refined parameters of the crystal structure are listed in Table 1 . Small amounts of the impurity phases $\mathrm{Er}_{2} \mathrm{O}_{3}(2.20 \mathrm{wt} \%)$ and $\mathrm{Cu}(0.15 \mathrm{wt} \%)$ were detected and were taken into consideration during refinement.

\subsection{Magnetic structure}

The unit cell contains six Er atoms that are expected to carry localized magnetic moments. Two of these atoms occupy the $2 c$ site, namely, Er1_1 at $\left(0,0, \frac{1}{2}\right)$ and Er1_2 at $\left(\frac{1}{2}, \frac{1}{2}, \frac{1}{2}\right)$, the latter being related to the former by the centring translation $\mathbf{c}$ $=\left[\frac{1}{2}, \frac{1}{2}, 0\right]$. The remaining four Er atoms occupy the $4 i$ site, i.e. Er2_1 at $\left(x_{\mathrm{Er} 2}, 0, z_{\mathrm{Er} 2}\right)$ and Er2_2 at $\left(1-x_{\mathrm{Er} 2}, 0,1-z_{\mathrm{Er} 2}\right)$, together with Er2_3 and Er2_4, whose positions are obtained by applying the centring translation $\mathbf{c}$ to the positions of Er2_1 and Er2_2.

In the neutron diffraction experiment, Bragg reflections of magnetic origin have been shown to appear as the sample temperature decreases. Fig. 1(b) presents a difference pattern corresponding to the magnetic contribution to the neutron diffraction pattern collected at $3.7 \mathrm{~K}$. The difference pattern has been obtained by subtracting the paramagnetic data taken at $7.9 \mathrm{~K}$ from those recorded at $3.7 \mathrm{~K}$. A numerical analysis of the positions of the Bragg reflections of magnetic origin
Table 2

Basis vectors (BV) of the allowed irreducible representations (IR) for $\mathbf{k}_{1}=$ $\left[0,0, \frac{1}{2}\right]$ and the $2 c$ Wyckoff site of space group $C 2 / m$.

The refined values $\left(C_{i}\right)$ denote contributions of a particular BV to the Fourier coefficients characterizing the magnetic structure. The results were obtained from Rietveld refinement of the following difference neutron diffraction patterns: 3.7-7.9 K (Fig. $1 b$ ) and 1.4-7.9 K (Fig. 1c). E9 diffractometer, with $\lambda$ $=2.815 \AA$.

\begin{tabular}{lllllll}
\hline & \multicolumn{2}{c}{ IR2 } & & IR4 & & \\
\cline { 2 - 3 } $\mathbf{k}_{1}=\left[0,0, \frac{1}{2}\right]$ & $\mathrm{BV} 1$ & $\mathrm{BV} 2$ & & $\mathrm{BV} 1$ & & \\
\hline Er1_1 $(2 c)$ at $\left(0,0, \frac{1}{2}\right):$ & 100 & 001 & 010 & & \\
\hline & $\begin{array}{l}C 1 \\
\left(\mu_{\mathrm{B}}\right)\end{array}$ & $\begin{array}{l}C 2 \\
\left(\mu_{\mathrm{B}}\right)\end{array}$ & $\begin{array}{l}C 1 \\
\left(\mu_{\mathrm{B}}\right)\end{array}$ & $\begin{array}{l}\mu_{\text {tot }} \\
\left(\mu_{\mathrm{B}}\right)\end{array}$ & $\begin{array}{l}R_{\text {magn }} \\
(\%)\end{array}$ \\
\hline 3.7 K & - & - & $8.86(7)$ & $8.86(7)$ & 8.52 \\
Er1_1 & - & - & $8.91(8)$ & $8.91(8)$ & 7.59 \\
$1.4 \mathrm{~K}$ & - & - & &
\end{tabular}

reveals antiferromagnetic order described by the propagation vector $\mathbf{k}_{1}=\left[0,0, \frac{1}{2}\right]$. In order to find physically valid magnetic structure models, a symmetry analysis has been performed. As the Rietveld refinement shows that this antiferromagnetic order is limited solely to the erbium moments on the $2 c$ site, the symmetry analysis of this site is presented in detail. The $\mathbf{k}_{1}$ propagation vector is equivalent to $-\mathbf{k}_{1}$ and is the only member of the star, while taking into account the space group $C 2 / m$. The Er1_1 and Er1_2 magnetic moments belong to one orbit, i.e. their directions and magnitudes are interrelated by symmetry. The basis vectors of the allowed irreducible representations for $\mathbf{k}_{1}=\left[0,0, \frac{1}{2}\right]$ and the $2 c$ Wyckoff site of space group $C 2 / m$ are listed in Table 2 . The naming of the

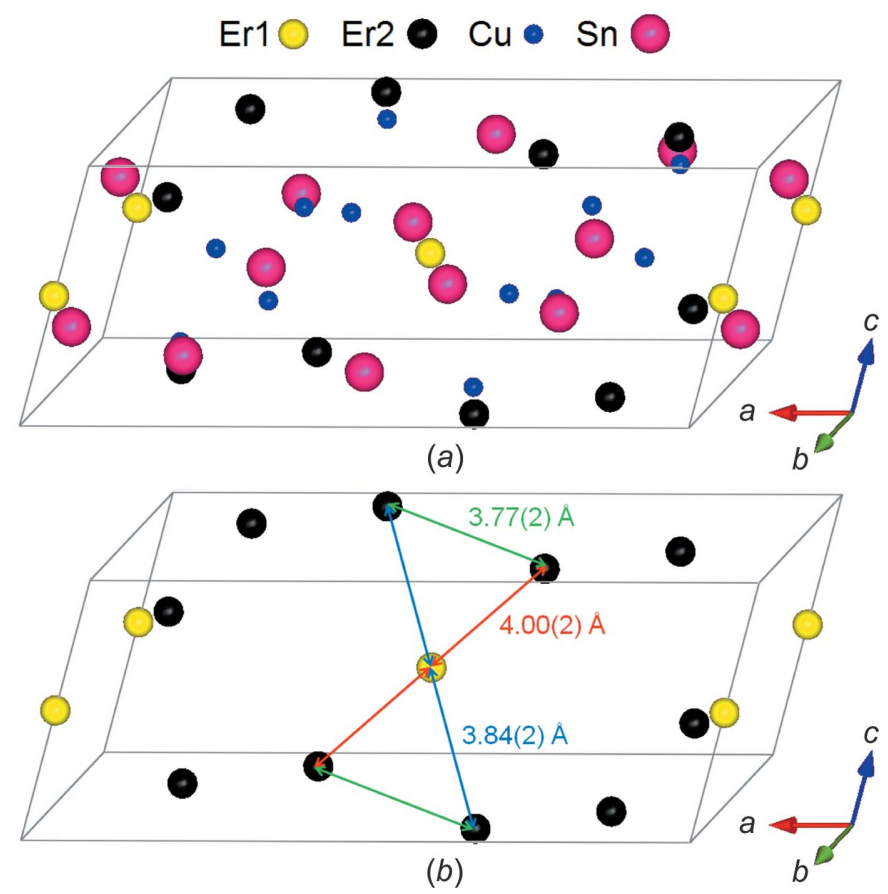

Figure 2

(a) The unit cell of $\mathrm{Er}_{3} \mathrm{Cu}_{4} \mathrm{Sn}_{4}$ in monoclinic space group C2/m. (b) The distribution of the $\mathrm{Er}$ atoms in space. The interatomic distances are indicated. 
representations and basis vectors follows the output of the BasIreps program from the FullProf package (RodríguezCarvajal, 1993). The best fit to the experimental data has been obtained for the Er1_1 and Er1_2 magnetic moments parallel to the $b$ axis (BV1 of IR4) and coupled ferromagnetically within one unit cell, while those in the neighbouring cells along the [001] direction are oriented antiparallel. Therefore, the magnetic unit cell is doubled along the c direction with respect to the crystal one [see Fig. 3(a)]. The Er magnetic moments on the $2 c$ site are found to be $8.86(7) \mu_{\mathrm{B}}$ at $3.7 \mathrm{~K}$, while the Er moments on the $4 i$ site remain disordered at that temperature.

As the temperature decreases further, additional Bragg peaks of magnetic origin appear in the diffraction pattern. Fig. 1(c) presents a magnetic contribution to the neutron diffraction pattern collected at $1.4 \mathrm{~K}$. Apart from the reflections related to the antiferromagnetic order on the $2 c$ site, which remains the same as described above, two additional groups of reflections can be identified: the high-intensity ones, which correspond to the magnetic ordering on the $4 i$ Er site, and the low-intensity ones, which have been identified as coming from the antiferromagnetic order in the $\mathrm{Er}_{2} \mathrm{O}_{3}$ impurity phase (Moon et al., 1968). The high-intensity reflec-

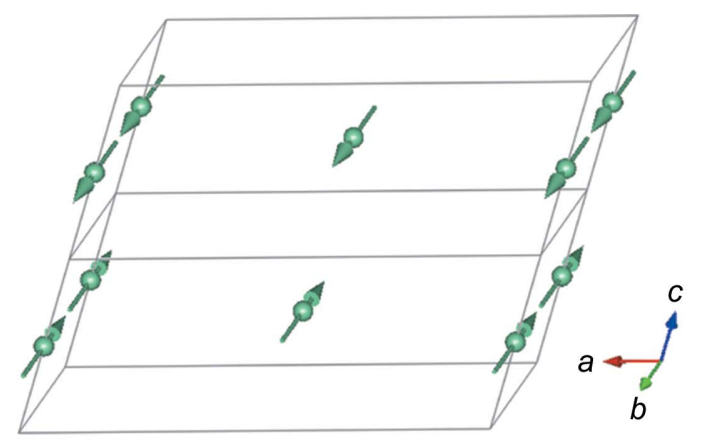

(a)

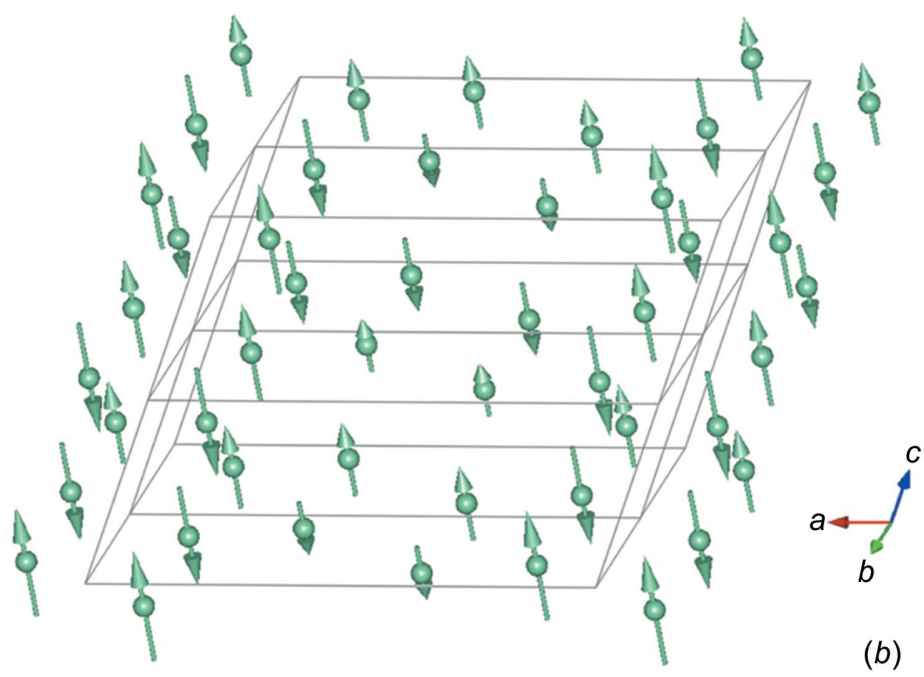

(b)

) tions can be described by the propagation vector $\left[0, k_{y}, \frac{1}{2}\right]$, where $k_{y}=0.4667$ (1) at $1.4 \mathrm{~K}$. However, a better fit is obtained while using $\mathbf{k}_{2}=\left[1,0.4667\right.$ (1), $\left.\frac{1}{2}\right]$ and that is why the magnetic structure related to the latter vector is described below. It is worth noting that these two vectors are not equivalent due to the presence of the centring translation $\mathbf{c}=$ $\left[\frac{1}{2}, \frac{1}{2}, 0\right]$. The $\mathbf{k}_{2}$ propagation vector forms a star together with $-\mathbf{k}_{2}$, while all Er magnetic moments occupying the $4 i$ site (i.e. from Er2_1 to Er2_4) belong to one orbit. The theory predicts two one-dimensional irreducible representations, each of them appearing three times as listed in Table 3. The best fit has been obtained for a sine-modulated antiferromagnetic structure with Er magnetic moments lying in the $a c$ plane and forming a right angle with the $a$ axis [the refinement provides an angle of $89(3)^{\circ}$ ] (Fig. 3b). The structure corresponds to a linear combination of the BV1 and BV3 basis vectors of IR2. As the crystallographic axes form a non-Cartesian coordinate system, the amplitude of the modulation can be calculated using the equation $\mu_{\mathrm{A}}=\left[(C 1)^{2}+(C 3)^{2}+2(C 1)(C 3) \cos \beta\right]^{1 / 2}$, where $C 1$ and $C 3$ are the parameters obtained from refinement. The amplitude of the modulation is found to be 8.7 (1) $\mu_{\mathrm{B}}$ at $1.4 \mathrm{~K}$.

Thermal evolution of the $\mathrm{Er}_{3} \mathrm{Cu}_{4} \mathrm{Sn}_{4}$ neutron diffraction pattern is presented in Fig. 4(a). Fig. 4(b) shows the refined
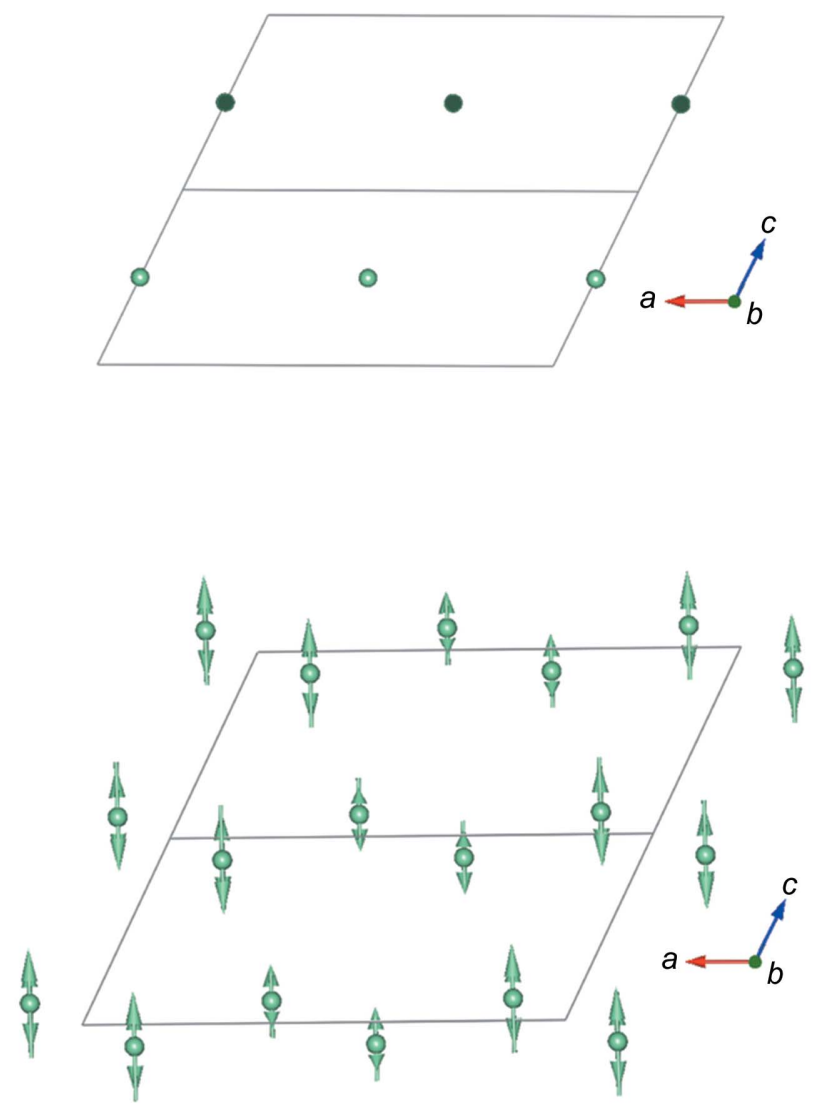

Figure 3

(a) Magnetic unit cell related to the commensurate antiferromagnetic order of the Er magnetic moments in the $2 c$ site $\left(\mathbf{k}_{1}=\left[0,0, \frac{1}{2}\right]\right)$. (b) Incommensurate sine-modulated magnetic structure formed by the Er moments in the $4 i$ site $\left(\mathbf{k}_{2}=\left[1,0.4667(1), \frac{1}{2}\right]\right)$. As the magnetic unit cell cannot be defined in the latter case, the figure presents a selected volume that is sufficient to demonstrate the basic pattern of this structure. The left-hand side of the figure shows a perspective view while the right-hand side presents its projection on the $a c$ plane. 
Table 3

Basis vectors $(\mathrm{BV})$ of the allowed irreducible representations (IR) for $\mathbf{k}_{2}=[1$, 0.4667 (1), $\left.\frac{1}{2}\right]$ and the $4 i$ Wyckoff site of the space group $C 2 / m$.

The refined values $\left(C_{i}\right)$ denote contributions of a particular BV to the Fourier coefficients characterizing the magnetic structure. The results were obtained from Rietveld refinement of the difference neutron diffraction pattern: 1.4-7.9 K (Fig. 1c). E9 diffractometer, with $\lambda=$ $2.815 \AA$.

\begin{tabular}{|c|c|c|c|c|c|c|c|c|}
\hline \multirow[b]{2}{*}{$\mathbf{k}_{2}=\left[1,0.4667(1), \frac{1}{2}\right]$} & \multicolumn{3}{|c|}{ IR1 } & \multicolumn{3}{|c|}{ IR2 } & & \\
\hline & BV1 & BV2 & BV3 & BV1 & $\mathrm{BV} 2$ & BV3 & & \\
\hline \multirow{3}{*}{$\begin{array}{l}\text { Er2_1 }(4 i) \text { at }\left(x_{\mathrm{Er} 2}, 0, z_{\mathrm{Er} 2}\right) \\
\operatorname{Er2} 22(4 i) \text { at }\left(1-x_{\mathrm{Er} 2}, 0,1-z_{\mathrm{Er} 2}\right)\end{array}$} & 100 & 010 & 001 & 100 & 010 & 001 & & \\
\hline & 100 & $0-10$ & 001 & -100 & 010 & $00-1$ & & \\
\hline & $\begin{array}{l}C 1 \\
\left(\mu_{\mathrm{B}}\right)\end{array}$ & $\begin{array}{l}C 2 \\
\left(\mu_{\mathrm{B}}\right)\end{array}$ & $\begin{array}{l}C 3 \\
\left(\mu_{\mathrm{B}}\right)\end{array}$ & $\begin{array}{l}C 1 \\
\left(\mu_{\mathrm{B}}\right)\end{array}$ & $\begin{array}{l}C 2 \\
\left(\mu_{\mathrm{B}}\right)\end{array}$ & $\begin{array}{l}C 3 \\
\left(\mu_{\mathrm{B}}\right)\end{array}$ & $\begin{array}{l}\mu_{\text {tot }} \\
\left(\mu_{\mathrm{B}}\right)\end{array}$ & $\begin{array}{l}R_{\operatorname{magn}} \\
(\%)\end{array}$ \\
\hline $1.4 \mathrm{~K}$ & & & & & & & & 9.85 \\
\hline Er2_1 & - & - & - & $4.3(4)$ & - & $9.6(2)$ & 8.7 (1) & \\
\hline Er2_2 & - & - & - & $4.3(4)$ & - & $9.6(2)$ & $8.7(1)$ & \\
\hline
\end{tabular}

diffraction studies reported in this work confirm the low-temperature monoclinic structure in both the paramagnetic and magnetically ordered state. The refined crystal structure parameters are in good agreement with previously reported data.

The Er magnetic moments are found to order antiferromagnetically below about $6 \mathrm{~K}$ ( $2 c$ site) and below about $2 \mathrm{~K}$ ( $4 i$ site). These temperatures are in agreement with previously reported anomalies in the magnetic susceptibility. The anomalies were observed at 5.8 and $2.2 \mathrm{~K}$ (Ryan et al., 2004) or at 6.0 and $2.2 \mathrm{~K}$ (Wawrzyńska et al., 2003). An additional anomaly reported at $3.6 \mathrm{~K}$ (Ryan et al., 2004; Wawrzyńska et al., 2003) can be attributed to the antiferromagnetic ordering in the $\mathrm{Er}_{2} \mathrm{O}_{3}$ impurity phase (Moon et al., 1968). A small amount (2.20 wt \%) of $\mathrm{Er}_{2} \mathrm{O}_{3}$ has been found in the sample reported in this work, but the same

values of the Er magnetic moment on the $2 c$ site $\left(\mathbf{k}_{1}=\left[0,0, \frac{1}{2}\right]\right)$, together with the amplitude of the modulation of the incommensurate magnetic structure formed by the Er moments on the $4 i$ site $\left(\mathbf{k}_{2}=\left[1,0.4667(1), \frac{1}{2}\right]\right.$ at $\left.1.4 \mathrm{~K}\right)$. According to the Rietveld refinement, the incommensurate component of the magnetic structure disappears at about $2 \mathrm{~K}$, while the commensurate one persists to about $6 \mathrm{~K}$.

\section{Discussion}

$\mathrm{Er}_{3} \mathrm{Cu}_{4} \mathrm{Sn}_{4}$ was originally reported to crystallize in the orthorhombic $\mathrm{Gd}_{3} \mathrm{Cu}_{4} \mathrm{Ge}_{4}$-type crystal structure at room temperature (Thirion et al., 1983). Further studies showed that the orthorhombic structure transforms at 262 (2) K into its monoclinically distorted variant, the $\mathrm{Tm}_{3} \mathrm{Cu}_{4} \mathrm{Sn}_{4}$-type crystal structure (Cadogan et al., 2014). The high-resolution neutron impurity phase was probably present in the previously investigated samples.

The Er moments in the $2 c$ sublattice order in a collinear commensurate antiferromagnetic structure with magnetic moments parallel to the $b$ axis and the magnetic unit cell doubled along the [001] direction with respect to the crystal one $\left(\mathbf{k}_{1}=\left[0,0, \frac{1}{2}\right]\right)$. The same magnetic structure is observed in isostructural $\mathrm{Tm}_{3} \mathrm{Cu}_{4} \mathrm{Sn}_{4}$ (Baran et al., 2013).

Collinear incommensurate antiferromagnetic order, related to $\mathbf{k}_{2}=\left[1, k_{y}, \frac{1}{2}\right.$ ] [where $k_{y}=0.4667$ (1) at $1.4 \mathrm{~K}$ ], develops in the $4 i$ sublattice below the Néel temperature $T_{\mathrm{N} 2}$, which is lower than that characterizing the ordering of the magnetic moments in the $2 c$ sublattice $\left(T_{\mathrm{N} 1}\right)$. The magnetic moments lie in the $a c$ plane and are perpendicular to the $a$ axis (they are inclined at an angle of about $26^{\circ}$ to the $c$ axis). This magnetic structure differs from that of isostructural $\mathrm{Tm}_{3} \mathrm{Cu}_{4} \mathrm{Sn}_{4}$, where

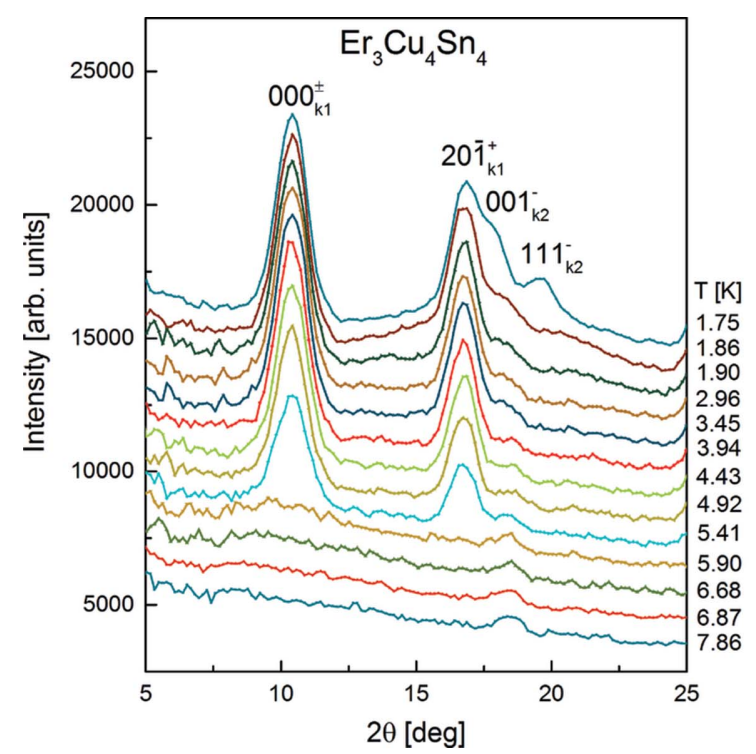

(a)

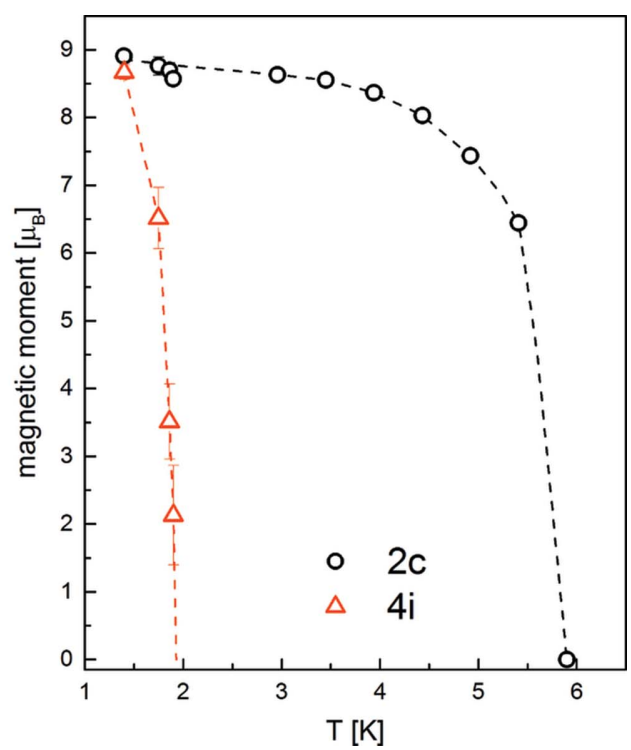

(b)

Figure 4

(a) Thermal evolution of the $\mathrm{Er}_{3} \mathrm{Cu}_{4} \mathrm{Sn}_{4}$ neutron diffraction pattern, based on the data collected between 1.75 and $7.86 \mathrm{~K}$. The $2 \theta$ range from 5 to $25^{\circ}$ is shown (E6 diffractometer, $\lambda=2.432 \AA$ ). (b) Temperature dependence of the Er magnetic moments located in the $2 c\left(\mathbf{k}_{1}=\left[0,0, \frac{1}{2}\right]\right)$ and $4 i\left(\mathbf{k}_{2}=[1\right.$, $\left.\left.0.4667(1), \frac{1}{2}\right]\right)$ sites. In the latter case, the amplitude of modulation is presented as the magnetic structure is incommensurate. 


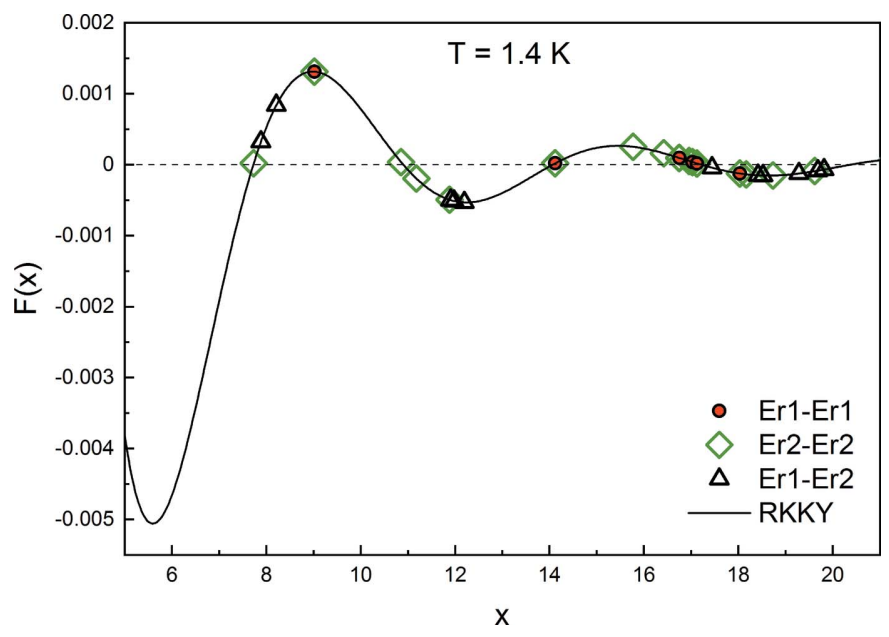

Figure 5

$F(x)$ function predicted by the Ruderman-Kittel-Kasuya-Yosida (RKKY) model, where $x=2 k_{F} r$ (see main text for details). The open symbols indicate the $\mathrm{Er}-\mathrm{Er}$ interatomic distances in $\mathrm{Er}_{3} \mathrm{Cu}_{4} \mathrm{Sn}_{4}$, calculated using the refined data from Table 1 . The Fermi vector $k_{F}$ is estimated following the free electron Fermi gas model (Kittel, 1996).

the magnetic moments are found to be parallel to the $c$ axis and the propagation vector is reported as $\left[\frac{1}{4}, 0.4739(1), 0\right]$ at $1.5 \mathrm{~K}$, indicating an extra modulation along the $a$ axis (Baran et al., 2013).

As presented above, the $2 c$ magnetic moments in the ordered state are parallel to the $b$ axis in both isostructural $R_{3} \mathrm{Cu}_{4} \mathrm{Sn}_{4}(R=$ Er and $\mathrm{Tm})$, while the $4 i$ moments lie in the $a c$ plane and are parallel $(R=\mathrm{Tm})$ or almost parallel $(R=\mathrm{Er})$ to the $c$ axis. Such a behaviour can be attributed to the influence of the Crystalline Electric Field (CEF) as the local point symmetry is different for the $2 c$ and $4 i$ sites, namely it is $2 / m$ and $m$, respectively. Another distinct feature is the difference in ordering temperatures: the $2 c$ magnetic moments order at higher temperatures than the $4 i$ ones. As the Er-Er interatomic distances are long enough to exclude direct interactions (the shortest distance is about $3.8 \AA$; see Table 1), the ordering of magnetic moments is expected to be driven by the indirect interactions of the Ruderman-Kittel-KasuyaYosida (RKKY) type. The RKKY model predicts that the exchange integral is proportional to the function $F(x)=$ $(\sin x-x \cos x) / x^{4}$, where $x=2 k_{F} r$ and $k_{F}$ refers to the Fermi vector, while $r$ refers to the interatomic distance (Blundell,
2001). Fig. 5 shows the $F(x)$ function compared to the $\mathrm{Er}-\mathrm{Er}$ interatomic distances in $\mathrm{Er}_{3} \mathrm{Cu}_{4} \mathrm{Sn}_{4}$. It is apparent that the exchange integral between the nearest neighbours (NN) in the Er2 sublattice is much smaller than the exchange integrals between the $\mathrm{NN}$ in the Er1 sublattice, as well as the NN belonging to different sublattices. As a result, the interactions in the Er1 sublattice are stronger and the corresponding Néel temperature is higher than that of the Er2 sublattice.

\section{Acknowledgements}

The kind hospitality and financial support extended to two of the authors (SB and AS) by the Helmholtz-Zentrum Berlin für Materialien und Energie (HZB) is gratefully acknowledged. The open-access publication of this article was funded by the Priority Research Area SciMat under the program 'Excellence Initiative - Research University' at the Jagiellonian University in Krakow.

\section{References}

Baran, S., Kaczorowski, D., Szytuła, A., Gil, A. \& Hoser, A. (2013). J. Phys. Condens. Matter, 25, 066012.

Blundell, S. (2001). In Magnetism in Condensed Matter. New York: Oxford University Press Inc.

Cadogan, J. M., Pérez, S. M., Cobas, R., Ryan, D. H., Lora-Serrano, R., Figueira, M. J. S. \& Yokaichiya, F. (2014). Intermetallics, 55, 123128.

Hanel, G. \& Nowotny, H. (1970). Monatsh. Chem. 101, 463-468.

Kittel, C. (1996). In Introduction to Solid State Physics. New York: John Wiley \& Sons Inc.

Moon, R. M., Koehler, W. C., Child, H. R. \& Raubenheimer, L. J. (1968). Phys. Rev. 176, 722-731.

Rieger, W. (1970). Monatsh. Chem. 101, 449-462.

Rodríguez-Carvajal, J. (1993). Phys. B Condens. Matter, 192, 5569.

Romaka, L., Romaka, V. V. \& Davydov, V. (2008). Chem. Met. Alloys, 1, 192-197.

Ryan, D. H., Cadogan, J. M., Gagnon, R. \& Swainson, I. P. (2004). J. Phys. Condens. Matter, 16, 3183-3198.

Ryan, D. H., Cadogan, J. M., Voyer, C. J., Napoletano, M., Riani, P. \& Cranswick, L. M. D. (2010). Mod. Phys. Lett. B, 24, 1-28.

Szytuła, A., Wawrzyńska, E. \& Zarzycki, A. (2007). J. Alloys Compd, 442, 200-202.

Thirion, F., Steinmetz, J. \& Malaman, B. (1983). Mater. Res. Bull. 18, $1537-1542$.

Wawrzyńska, E., Hernandez-Velasco, J., Penc, B., Sikora, W., Szytuła, A. \& Zygmunt, A. (2003). J. Phys. Condens. Matter, 15, 5279-5296. 